\title{
Management of Acute Native Joint Bacterial Arthritis in Adults in 2020 - A Short Narrative, Practical State-of-the-Art Review
}

\author{
Alexandre Ansorge ${ }^{1}$, Farah Selman ${ }^{1}$ and Ilker Uçkay ${ }^{1,2 *}$ \\ ${ }^{1}$ Department of Orthopedics, Balgrist University Hospital, Zurich, Switzerland \\ ${ }^{2}$ Infectiology, Balgrist University Hospital, Zurich, Switzerland \\ *Corresponding Author: Ilker Uçkay, Professor, Balgrist University Hospital, Zurich, \\ Switzerland.
}

Received: December 16, 2020

Published: December 29, 2020

(C) All rights are reserved by Ilker Uçkay., et al.

\begin{abstract}
Native joint bacterial arthritis is a common infection among adults and children. A solely conservative management, without any articular drainage/lavage, increases the risk of recurrence. In contrast, the type of initial lavage/drainage can be surgical (arthrotomy or arthroscopy) or non-surgical (iterative arthrocenteses). Up to date, no superiority has been shown for any of these approaches in relation to recurrence risk and postinfectious mechanical damage. Furthermore, an initial synovectomy, or the number of iterative drainages does not influence outcome in most cases. Nowadays, an antibiotic regimen of three to four weeks, with early oral therapy, is standard in most settings of the world. In arthritis cases involving the hand and wrist, a shorter systemic antibiotic treatment such as two weeks is sufficient. The outcome of infection is impacted by of mechanical sequelae in up to $40 \%$ of cases. These sequels are predominantly joint stiffness and/or osteoarthritis, which are difficult to treat.
\end{abstract}

Keywords: Native Arthritis; Antibiotics; Surgical Drainage; Management; Narrative Review

\section{Introduction}

The clinical appearance of native joint bacterial arthritis are very close to those of any non-infectious arthritis, such as viral, reactive, crystal-induced, or auto-immune arthritis. Yet, bacterial arthritis should be diagnosed and treated early in the process, since enzymes liberated by bacterial pathogens or reacting granulocytes risk to damage the bradytroph cartilage. Available literature about bacterial arthritis is composed of plenty expert opinions and reviews, while original research articles are less frequent [1]. In this narrative review, we focus on the current "lege artis" clinical management of native joint bacterial arthritis based on available published evidence.

Epidemiology, origins and causative pathogens

The annual incidence of native joint bacterial arthritis in wealthy countries varies between $2-10$ cases per 100,000 persons
[2-4] as well as patient charts with a discharge diagnosis of septic arthritis (International Statistical Classification of Diseases and Related Health Problems (ICD. Most septic arthritis is monoarticular. Indeed, only 2.7 to $15 \%$ present as a polyarticular arthritis [5-10] the proportion of septic arthritis cases involving people who inject drugs (PWID. The majority of septic arthritis cases occur spontaneously or are believed to be secondary to an hematogenous spread of bacteria from a skin lesion into the joint [2-4] as well as patient charts with a discharge diagnosis of septic arthritis (International Statistical Classification of Diseases and Related Health Problems (ICD. Spontaneous infection involves predominantly the major weight-bearing joints. Indeed, the knee is affected most of the time, followed in decreasing frequency by the hip, the elbow, the hand, the ankle, the wrist, the sternoclavicular and finally the sacroiliac articulations [6]. Globally, Staphylococcus aureus is the most preva- 
lent pathogen in 50-60\%, followed by streptococci (16\%-17\%), gram-negative rods $(5 \%-15 \%)$, and anaerobes $(<1 \%)[4,5]$ clinical pattern and outcome. We reviewed the literature from 1945 to 2010 with an emphasis on post-traumatic cases. We retrieved 14 large-scale epidemiological surveys without detailed stratification regarding the origin of septic arthritis (3,340 episodes. In contrast to spontaneous adult cases where Staphylococcus aureus is most prevalent, in infants the most encountered bacteria is a gram-negative rod named Kingella kingae [11]we aimed to contrast the bacteriologic epidemiology of osteoarticular infections (OAIs).

\section{Diagnosis}

Acute native joint arthritis usually presents with a swollen articulation, local erythema and heat, and worsening arthralgia. Most patients are transiently febrile. High fever spikes are uncommon, unless there is a synchronous bacteraemia [12]. One should in particular look for indices of remote infections like for instance endocarditis, respiratory, urinary tract, sexually-transmitted and skin infections. In sexually-active patients without adequate protection, Neisseria gonorrhoeae, is still quite prevalent [13]. Risk factors such as autoimmune rheumatic disease and intravenous drug abuse should also be looked for. Interestingly, patients infected with the human immunodeficiency virus don't have an increased risk to develop septic arthritism unless they are concomitant intravenous drug abusers [1]. If the detailed anamnesis and the general physical exam fail to identify a remote origin of the septic arthritis, we generally don't recommend to perform advanced diagnostic tests to force the recognition of the origin of the infection because the antibiotic treatment usually cure it, so the expenses for endoscopy and complex radiologic exams can be avoided [14]. One should also be aware that septic arthritis may coincide with crystalline arthritis $[15,16] 227$ with underlying arthroplasties and 69 with gout or other crystals in synovial fluid. In such cases, it remains unclear if the crystalline inflammation is prejudicial in the acute phase. However, evidence exists in favor of the use of anti-crystalline treatment like colchicine in case of an acute gout developing just after the drainage of a joint for septic arthritis [1]. The gold standard for diagnosing bacterial arthritis is the objectivation of an identical pathogen in at least two samples of synovial fluid or intraarticular tissue biopsy, or a clinical arthritis associated with synchronous positive blood cultures that aren't considered as contamination. Blood cultures should be performed in case of fever and shivering, considering that $50 \%$ of them turn positive [2].
However, a culture-negative bacterial arthritis may also occur. Risk factors for culture-negative cases are a low inoculum, a prior antibiotic exposure, an inappropriate culture media, fastidious bacteria, or a prolonged transport time to the laboratory favor negative-culture results [17]1167 (43\%. A clear-cut definition of culture-negative bacterial arthritis doesn't exist. It is usually diagnosed in cases showing a typical clinical presentation with suggestive pathologic synovial fluid laboratory results (granulocyte proportion of $>90 \%$ and/or leukocyte count over 50 ' 000 cells $/ \mu \mathrm{l}$ ), and no intraarticular crystals. Polymerase chain techniques (PCR) shouldn't be routinely used, because eubacterial PCR is quite expensive and seldom of benefit. Its sensitivity is lower than that of classical culture. Moreover, it doesn't give clues about antibiotic resistance and in the case of a polymicrobial infection its interpretation is hazardous [1]. In particular clinical situations suggesting an infection by one of the following pathogens, specific PCR is however recommended: Kingella kingae, Brucella spp, Borrelia spp, Coxiella burnetii, Neisseria gonorrhoeae, Bartonella henselae, Mycobacterium tuberculosis, or Mycobacterium ulcerans are such examples [1].

Laboratory inflammatory markers can be tested systemically (i.e. in the blood) and intraarticularly. High white blood cell counts and C-reactive protein concentrations in the blood serum aren't proving a bacterial origin of an arthritis regardless of the used cutoff. Indeed, these inflammatory markers presumably rather reflect the pathogen's virulence and the synchronous bacteremia than the local intraarticular state $[18,19]$. Therefore, they are also useless in the follow up, concerning decision making about the duration of antibiotic treatment or the indication of repeated joint drainage/lavage. It is still important to know that serum procalcitonin concentration is often low in the case of a localized septic arthritis lacking systemic inflammatory signs $[19,20]$. Usually, synovial leukocyte counts and neutrophil proportions are determined. These tests must however be interpretated with caution, as no cut-off values have been shown to be diagnostic for bacterial arthritis. Concerning synovial leukocyte counts, many experts consider a number of $>50,000$ cells $/ \mu$ l as predictive for a bacterial arthritis [21]. However, there is equally no consensus about this threshold value and other authors set it for example at $>100,000$ cells/ $\mu$ [ [22] not the surrounding soft tissues. The most common causes of monoarthritis are crystals (i.e., gout and pseudogout. Concerning the neutrophil proportion in the synovial fluid, a literature review including 6242 cases showed a three-fold probability for septic arthritis in patients with a neutrophil proportion of $>90 \%$ in comparison to 
others [23]prompt identification and treatment of septic arthritis can substantially reduce morbidity and mortality. \nOBJECTIVE: To review the accuracy and precision of the clinical evaluation for the diagnosis of nongonococcal bacterial arthritis.\nDATA SOURCES: Structured PubMed and EMBASE searches (1966 through January 2007. In future, other intra-synovial marker tests may appear [24]. For instance, research about intraarticular lactic acid dosing is warranted [25] as well as in 12 cases of gonococcal arthritis (mean $27 \mathrm{mg} / 100 \mathrm{ml}$.

Classical direct microscopic exams using Gram and acridinorange staining have been abandoned by many centers as its cost-benefit analyses are unsatisfying. The low sensitivity of direct microscopy isn't surprising as a low intraarticular inoculum is enough to trigger a significant arthritis. The results of Cunningham., et al. illustrate well the low sensitivity of direct microscopy [16]227 with underlying arthroplasties and 69 with gout or other crystals in synovial fluid. In their series of 500 adult patients with suspected septic arthritis, they detected pathogens in only $29 \%$ of cases. When considering only the culture-positive cases (gold standard), they still yielded a low sensitivity of $37 \%$.

\section{Therapy}

The treatment of native joint septic arthritis in adults is mainly based on experts' opinion and on regional habits, because evidence is lacking. As a consequence, the treatments vary among many parts of the world. However, it is generally articulating around two principles, namely lavage/drainage of the affected joint(s) and synchronous systemic antibiotic therapy [18]. Available evidence suggests a higher recurrence risk if only one of these two principles is used for treatment. For instance, a retrospective work found a 21-fold increase of the recurrence risk in case of a strict conservative antibiotic management without any lavage/ drainage [18]. A strict surgical management with iterative lavages/ drainages is in turn impacted by a higher probability of deformity and consecutive osteitis [1].

\section{Drainage}

Acute bacterial arthritis is usually considered as an absolute emergency, necessitating immediate lavage/drainage of the af- fected joint. This opinion is relying basically on animal studies, while available clinical studies in humans fail to support it $[1,23]$ caused by autoimmunity, immune complexes, crystals, or cartilage damage, is clinically similar to native septic arthritis. The large microbiological spectrum of joint infection is one of the particularities distinguishing arthritis from other orthopedic infections such as osteomyelitis or implant-related infections. The current literature often defines orthopedic infections as "osteoarticular," suggesting that diagnosis and treatment of bone and joint infection would be similar. Septic arthritis, osteomyelitis, and orthopedic implant-associated infections are different in nature, epidemiology, therapy, and outcome. This chapter underlines the particularities of native joint arthritis with an emphasis on diagnosis, epidemiology, treatment, and some prevention aspects before planned joint interventions. Most cases of primary septic arthritis and surgical site infections (SSIs. For instance, Lauper., et al. retrospectively analyzed the long-term outcome of over 200 native joint adult bacterial arthritis cases in relation to the delay of surgical drainage since the hospital admission [26]requiring drainage within hours, including during night, weekend or holiday shifts. However, there are few data supporting the need for the disruption caused by this degree of urgency. \nMETHODS: We performed a retrospective review of all adult patients seen in our medical center from 1997-2015 with cultureproven septic arthritis and noted the epidemiology of sequelae, and their possible association with a delay in surgical drainage. nRESULTS: Of 204 septic arthritis episodes, 46 (23\%. Interestingly, no outcome difference could be shown between a delay of less than 6 hours, 6-12 hours or even exceeding 24 hours. They also found a mean span of symptoms of 3 days ahead of hospital admission. Another study by Vispo-Seara., et al. found a time lapse between the onset of septic arthritis and arthroscopic lavage/drainage of over 2 weeks as being associated with advanced cartilage damage [27]. Balabaud., et al. have found a delay of less than 12 days until surgical drainage to be associated with healing of knee septic arthritis, as compared with a delay of 23 days associated with treatment failure [28].

Current literature indicate that any type of joint drainage is valid. The choice of drainage type is also depending on regional practice. While surgeons prefer arthrotomy or arthroscopy for drainage, many rheumatologists choose iterative arthrocentesis 
[1,29-31]. Concerning adult septic arthritis, no randomized clinical trials comparing surgical drainage to iterative arthrocentesis are available. Only retrospective reports are existing. For instance, Harada., et al. conducted a ten year retrospective single-center study to compare outcomes based on the taken management approach: medical (bedside closed-needle joint aspiration) versus surgical (arthrotomy/arthroscopy) [29]based on medical versus surgical management.\nMETHODS: A 10-year retrospective single-center study was conducted of patients admitted to a tertiary care hospital between January 1, 2006 and December 31, 2015 with a diagnosis of SA to compare outcomes based on the management approach taken: medical (bedside closed-needle joint aspiration. They evaluated outcomes were: the joint recovery, time to recovery, length of hospital stay, disposition to home versus rehabilitation unit, recurrence of arthritis in the same joint, and mortality. There was no statistically significant difference in long-term outcomes between the two groups at 12 months. Some surgeons plan a second look after the initial surgical drainage/lavage, based exclusively on the intraoperative visual aspect of the joint or on the fact that serum C-reactive protein concentrations aren't decreasing in further follow-up. This attitude isn't supported by evidence. We rather recommend to base the indication for a repeated surgical drainage according to the global clinical course.

\section{Antibiotic treatment}

The spectrum

Until the susceptibility results are available, the initial empirical antibiotic therapy in bacterial joint arthritis should cover the most frequent pathogens. After that, the initial regimen should be replaced by the narrowest possible spectrum. Methicillin-susceptible Staphylococcus aureus and streptococci should be covered at the beginning. In case of posttraumatic arthritis (e.g. bites or splinter injuries), Gram-negative bacteria have to be covered as well. In such cases, empirical intravenous (IV) administration of first- or second-generation cephalosporin or aminopenicillin is indicated. If community-acquired MRSA (Methicillin-resistant S. aureus) is suspected, vancomycin is indicated; respectively clindamycin or daptomycin, if an allergy to $\beta$-lactam antibiotics is present [32]. According to the expert opinion, empirical therapy starts intravenously [1]. All intravenous and the majority of oral antibiotics do penetrate enough the synovia and do reach concentrations exceeding the minimal inhibitory breakpoints of common pathogens $[33,34]$. In acute virulent arthritis early IV-application makes sense, but a switch to moral therapy is possible as soon as the clinical course is favorable. All non-beta-lactam antibiotics, as for example cotrimoxazole and quinolones are well-known for their good oral synovial bioavailability $[35,36]$ where, for at least the first 2-6 weeks, antibiotics should be administered intravenously, is more and more challenged in favor of an oral antibiotic treatment with selected agents from the start. There is no evidence that the total duration of antibiotic therapy for more than 4-6 weeks improves outcome, when compared with shorter regimens. Hopefully, the future will show randomized trials in the adult population, allowing optimal timing of surgical and medical therapy and sparing of unnecessary prescription, with concomitant development of antibiotic resistance. External advice from an expert team with combined surgeons and infectious disease physicians may help to reduce antibiotic consumption in a cost-effective way. Beta-lactam antibiotics have a low oral bioavailability and should therefore be avoided for the oral route. An intraarticular antibiotic administration is not indicated because of its potential cartilage toxicity [37] the most frequently used antiseptics are polyhexanide, hydrogen peroxide and taurolidine. The aim of this study was to examine the effects of these antiseptics on viability of human chondrocytes. Our hypothesis was that antiseptics and supplemental irrigation with sodium chloride lavage are less toxic on human chondrocytes than treatment with antiseptics only. Primary human chondrocytes were isolated and cultured from six donated human knee joints. Polyhexanide, hydrogen peroxide or taurolidine were added to the cultures. Toxicity analysis was performed by visualisation of cell structure using light microscopy and LDH activity. The determination of vital cells and total cell numbers of chondrocytes treated with antiseptics partly followed by irrigation with sodium chloride solution was performed by using Casy Cell-Counter. Light microscopic data revealed a defect in cell structure after addition of antiseptics. We showed a significant increase of LDH enzyme activity after the treatment with polyhexanide or taurolidine. After treatment with antiseptics followed by sodium chloride solution a significant increase of vital and total cell numbers resulted in comparison with the chondrocytes that were only treated with antiseptics. The data show that treatment with polyhexanid, hydrogen peroxide or taurolidine induces cell death of human chondroctes in vitro. 
The application of sodium chloride solution after the treatment with polyhexanide and hydrogen peroxide possibly has a protective effect on chondrocyte viability.

\section{Duration of antimicrobial therapy}

The optimal duration of post-drainage systemic antibiotic treatment is controversial. Often the indicated therapy might depend on the individual case. Some experts advocate a 3-4 weeks IV antibiotic therapy for staphylococci and Gram-negative pathogens, a 2-week IV therapy for streptococci, and an IV therapy exceeding four weeks for immune-compromised patients [2]. Nade., et al. recommend a 2 weeks IV therapy for streptococci [38]. Often, an IV therapy is recommended for the first 2 weeks, followed by a 2 -week oral therapy. Yet, this duration is currently considered as excessive for drained, adult native joints infections. Indeed, a total antibiotic treatment duration of 3-4 weeks is sufficient [33,39]. The antimicrobial treatment in bacterial hand and wrist arthritis is usually very short. Angly., et al. showed that no recurrence occurred in operated adult finger arthritis after antibiotics administered for a median duration of 2 days IV and 17 days orally [40] surgical technique, antimicrobial therapy and hand therapy. \nPATIENTS AND METHODS: Data of 31 patients with surgical treatment between 1993 and 2005 were screened and those from 29 patients with $>1$ year of follow-up were retrospectively analyzed. Surgical techniques were debridement, primary, secondary arthrodesis or temporary joint distraction with external fixation. In addition, all patients were treated with antibiotics and hand physiotherapy. The median follow-up of the 29 patients was 5.7 years. $\backslash$ nRESULTS: The most frequent cause was work injury (19/29. In another study including 101 hand arthritis cases and reporting similar outcome, the switch to oral therapy occurred 3-5 days postoperatively, which was recently confirmed in another narrative review [41]. It is not yet clear whether a short course of therapy is indicated in adult patients with large joint arthritis. Gjika., et al. recently issued a prospective-randomized study questioning the sufficient length of antimicrobial therapy in 154 patients with native joint bacterial arthritis [42] unblinded, randomised, non-inferiority study comparing either 2 or 4 weeks of antibiotic therapy after surgical drainage of native joint bacterial arthritis in adults. Excluded were implant-related infections, episodes without surgical lavage and episodes with a follow-up of less than 2 months. \nRESULTS: We enrolled 154 cases: 77 in the 4-week arm and 77 in the 2-week arm. Median length of intravenous antibiotic treatment was 1 and 2 days, respectively. The median number of surgical lavages was 1 in both arms. Recurrence of infection was noted in three patients $(2 \%$. One third of them suffered from medium to large joint infection. This study compared 2 weeks with 4 weeks of systemic targeted antibiotics after surgical drainage. The outcomes showed no differences regarding clinical remissions, adverse events or mechanical sequels. The median duration of initial IV-therapy was limited to 2 days. These results support the hypothesis that a short course of initial IV antibiotic administration is sufficient to treat adult native joint arthritis, similarly to other publications reporting pediatric cases [39].

\section{Outcomes}

Primary bacterial native joint arthritis is exceptionally fatal. If it is so, usually a severe underlying remote origin, such as endocarditis or a concomitant sepsis is usually responsible for the mortal outcome. Indeed, local infection remission frequently occurs in $97 \%$ in small joints, respectively in $90-95 \%$ in large joints $[18,42]$ to gather data for a prospective study on an optimized antibiotic treatment in adults with septic arthritis. \nMETHODS: This was a retrospective single-center study conducted for the period 19962008. \nRESULTS: A total of 169 episodes of septic arthritis in 157 adult patients (median age 63 years; 65 females. In contrast to the good infection control after eventually several drainages, the mechanical consequences are of major concern. Prospective randomized trials in adults with septic arthritis reported a frequency of mechanical sequels between $20 \%$ and $35 \%$, of which $15 \%$ required further surgical intervention [26]requiring drainage within hours, including during night, weekend or holiday shifts. However, there are few data supporting the need for the disruption caused by this degree of urgency. \nMETHODS: We performed a retrospective review of all adult patients seen in our medical center from 19972015 with culture-proven septic arthritis and noted the epidemiology of sequelae, and their possible association with a delay in surgical drainage.\nRESULTS: Of 204 septic arthritis episodes, 46 (23\%. Currently efforts are done to try to treat them by re-education, sensory-integrative therapy and eventual corrective surgery such as joint prostheses. 


\section{Conclusion}

Native joint bacterial arthritis is common. An articular drainage/lavage (arthrotomy or arthroscopy, or iterative arthrocentesis is always warranted. Up to date, no superiority has been shown for any of these approaches in relation to recurrence risk and postinfectious mechanical damage. Furthermore, an antibiotic regimen of three to four weeks, with early oral therapy, is standard. In arthritis cases involving the hand and wrist, a shorter systemic antibiotic treatment such as two weeks is sufficient. The outcome of infection is impacted by of mechanical sequelae, which are difficult to treat and need farther research for clinical improvement.

\section{Bibliography}

1. Uçkay I., et al. "Native Joint Arthritis". Bone and Joint Infections, John Wiley and Sons, Ltd. (2015): 77-91.

2. Geirsson AJ., et al. "Septic arthritis in Iceland 1990-2002: increasing incidence due to iatrogenic infections". Annals of the Rheumatic Diseases 67 (2008): 638-643.

3. Favero M., et al. "Septic arthritis: a 12 years retrospective study in a rheumatological university clinic". Reumatismo 60 (2008): 260-267.

4. Di Benedetto C., et al. "Post-traumatic septic arthritis". European Musculoskeletal Review 7 (2012): 38-42.

5. Ross JJ., et al. "Septic Arthritis and the Opioid Epidemic: 1465 Cases of Culture-Positive Native Joint Septic Arthritis From 1990-2018". Open Forum Infection Disease 7 (2020.

6. Weston V.C., et al. "Clinical features and outcome of septic arthritis in a single UK Health District 1982-1991". Annals of the Rheumatic Diseases 58 (1999): 214-219.

7. Kennedy N., et al. "Native Joint Septic Arthritis: Epidemiology, Clinical Features, and Microbiological Causes in a New Zealand Population". Journal of Rheumatology 42 (2015): 23922397.
8. Clerc 0., et al. "Adult native septic arthritis: A review of 10 years of experience and lessons for empirical antibiotic therapy". Journal of Antimicrobial and Chemotherapy 66 (2011): 1168-1173.

9. Kaandorp CJ., et al. "Incidence and sources of native and prosthetic joint infection: a community based prospective survey". Annals of the Rheumatic Diseases 56 (1997): 470-475.

10. Stutz G., et al. "Arthroscopic management of septic arthritis: stages of infection and results". Knee Surgery, Sports Traumatology, Arthroscopy 8 (2000): 270-274.

11. Samara E., et al. "Kingella kingae and Osteoarticular Infections”. Pediatrics (2019); 144.

12. Carpenter C.R., et al. "Evidence-based diagnostics: adult septic arthritis". Academic Emergency Medicine 18 (2011): 781-796.

13. Rice PA. "Gonococcal arthritis (disseminated gonococcal infection)". Infectious Disease Clinics of North America 19 (2005): 853-861.

14. Bouvet C., et al. "No need to search for the source of haematogenous arthroplasty infections". Swiss Medical Weekly 141 (2011).

15. Long B., et al. "Evaluation and Management of Septic Arthritis and its Mimics in the Emergency Department". Western Journal of Emergency Medicine 20 (2019): 331-341.

16. Cunningham G., et al. "Gram and acridine orange staining for diagnosis of septic arthritis in different patient populations". International Orthopaedics 38 (2014): 1283-1290.

17. Al-Mayahi M., et al. "Administration of antibiotic agents before intraoperative sampling in orthopedic infections alters culture results". Journal of Infection 71 (2015): 518-525.

18. Uçkay I., et al. "Short parenteral antibiotic treatment for adult septic arthritis after successful drainage". International Journal of Infectious Diseases 17 (2013): 199-205. 
19. Hügle T., et al. "Serum procalcitonin for discrimination between septic and non-septic arthritis". Clinical and Experimental Rheumatology 26 (2008): 453-456.

20. Uckay I., et al. "Postoperative serum pro-calcitonin and C-reactive protein levels in patients with orthopedic infections". Swiss Medical Weekly 140 (2010): 13124.

21. Brannan S.R., et al. "Synovial fluid analysis". Journal of Emergency Medicine 30 (2006): 331-339.

22. Siva C., et al. "Diagnosing acute monoarthritis in adults: a practical approach for the family physician". American Family Physician 68 (2003): 83-90.

23. Margaretten M.E., et al. "Does this adult patient have septic arthritis?" JAMA 297 (2007): 1478-1488.

24. Baillet A., et al. "Calprotectin discriminates septic arthritis from pseudogout and rheumatoid arthritis". Rheumatology (Oxford) 58 (2019): 1644-1648.

25. Brook I., et al. "Synovial fluid lactic acid. A diagnostic aid in septic arthritis". Arthritis and Rheumatology 21 (1978): 774779.

26. Lauper N., et al. "Native septic arthritis is not an immediate surgical emergency". Journal of Infection 77 (2018): 47-53.

27. Vispo Seara JL., et al. "Arthroscopic treatment of septic joints: prognostic factors". Archives of Orthopaedic and Trauma Surgery 122 (2002): 204-211.

28. Balabaud L., et al. "Results of treatment of septic knee arthritis: a retrospective series of 40 cases". Knee Surgery, Sports Traumatology, Arthroscopy 15 (2007): 387-392.

29. Harada K., et al. "Native Joint Septic Arthritis: Comparison of Outcomes with Medical and Surgical Management". South Medical Journal 112 (2019): 238-243.
30. Ravindran V., et al. "Medical vs surgical treatment for the native joint in septic arthritis: a 6-year, single UK academic centre experience". Rheumatology (Oxford) 48 (2009): 1320-1322.

31. Smith SP., et al. "Septic arthritis of the shoulder in children in Malawi. A randomised, prospective study of aspiration versus arthrotomy and washout". Journal of Bone and Joint Surgery. British 84 (2002): 1167-1172.

32. Liu C., et al. "Clinical practice guidelines by the Infectious Diseases Society of America for the treatment of methicillin-resistant Staphylococcus aureus infections in adults and children". Clinical Infection Disease 52 (2011): 18-55.

33. Thabit A.K., et al. "Antibiotic penetration into bone and joints: An updated review". International Journal of Infectious Diseases 8 (2019): 128-136.

34. Montange D., et al. "Penetration of daptomycin into bone and synovial fluid in joint replacement". Antimicrobial Agents and Chemotherapy 58 (2014): 3991-3996.

35. Uçkay I., et al. "Chronic osteomyelitis". Current Infectious Disease Reports 14 (2012): 566-575.

36. Schrenzel J., et al. "A randomized clinical trial to compare fleroxacin-rifampicin with flucloxacillin or vancomycin for the treatment of staphylococcal infection". Clinical Infection Disease 39 (2004): 1285-1292.

37. Röhner E., et al. "Toxicity of antiseptics against chondrocytes: What is best for the cartilage in septic joint surgery?" International Orthopaedics 35 (2011): 1719-1723.

38. Nade S. "Septic arthritis". Best Practice and Research: Clinical Rheumatology 17 (2003): 183-200.

39. Peltola H., et al. "Prospective, randomized trial of 10 days versus 30 days of antimicrobial treatment, including a short-term course of parenteral therapy, for childhood septic arthritis". Clinical Infection Disease 48 (2009): 1201-1210. 
40. Angly B., et al. "Septic arthritis of finger joints". Handchirurgie, Mikrochirurgie, plastische Chirurgie 39 (2007): 118-123.

41. Sendi P., et al. "Bone and joint infections of the hand". Clinical Microbiology and Infection 26 (2020): 848-856.

42. Gjika E., et al. "Two weeks versus four weeks of antibiotic therapy after surgical drainage for native joint bacterial arthritis: a prospective, randomised, non-inferiority trial". Annals of the Rheumatic Diseases 78 (2019): 1114-1121.

\section{Assets from publication with us}

- Prompt Acknowledgement after receiving the article

- Thorough Double blinded peer review

- Rapid Publication

- Issue of Publication Certificate

- High visibility of your Published work

Website: https://www.actascientific.com/

Submit Article: https://www.actascientific.com/submission.php

Email us: editor@actascientific.com

Contact us: +919182824667 